\title{
Un René Guillot inconnu : le romancier des années 1930
}

\section{Maria Chiara Gnocchi}

\section{Q OpenEdition}

\section{Journals}

\section{Édition électronique}

URL : http://journals.openedition.org/rief/1163

DOI : $10.4000 /$ rief. 1163

ISSN : 2240-7456

\section{Éditeur}

Seminario di filologia francese

\section{Référence électronique}

Maria Chiara Gnocchi, « Un René Guillot inconnu : le romancier des années 1930 », Revue italienne d'études françaises [En ligne], 6 | 2016, mis en ligne le 15 décembre 2016, consulté le 19 avril 2019 URL : http://journals.openedition.org/rief/1163 ; DOI : 10.4000/rief.1163

Ce document a été généré automatiquement le 19 avril 2019.

\section{(c) (i) (9)}

Les contenus de la RIEF sont mis à disposition selon les termes de la Licence Creative Commons Attribution - Pas d'Utilisation Commerciale - Pas de Modification 4.0 International. 


\title{
Un René Guillot inconnu : le romancier des années 1930
}

\author{
Maria Chiara Gnocchi
}

1 René Guillot (1900-1969) est un auteur renommé dans le domaine de la littérature pour la jeunesse, ou qui l'a été en tout cas, surtout dans les années 1960. Ses livres les plus célèbres ont été traduits dans une quinzaine de langues. On retiendra parmi d'autres Sirga la Lionne (1951, que Luc Besson a adapté au cinéma en 1993, sous le titre L'Enfant lion), CrinBlanc (1959, qui est par contre une novellisation du film d'Albert Lamorisse), Le Maître des éléphants (1960) et des séries comme Grichka ou Un petit chien. Ce qui est moins connu, c'est qu'au début de sa carrière, Guillot a écrit des romans non destinés à la jeunesse, qui couvrent presque exactement la période des années 1930. Il s'agit de six romans, dont le premier, La Grande Renaude, date de 1929 ; suivent Histoire d'un Blanc qui s'était fait nègre en 1932, Taillis en 1934, Ras el Gua en 1936, Vent de Norois en 1938 et Loaga en $1941^{1}$. Certains de ces textes - Histoire d'un Blanc qui s'était fait nègre en premier, mais Ras el Gua aussi mériteraient sûrement d'être redécouverts.

2 Après avoir brièvement présenté l'œuvre de Guillot dans son ensemble, je voudrais proposer des pistes de lecture pour ses romans des années 1930 afin d'expliquer pourquoi on gagnerait à les reconsidérer. Une attention particulière sera accordée au récit le plus complexe, Histoire d'un Blanc qui s'était fait nègre (dorénavant Histoire...). Je conclurai en m'interrogeant sur les raisons de l'oubli dans lequel ces textes ont sombré, et je crois que certaines de mes remarques peuvent s'étendre aux œuvres d'autres écrivains de la même période.

\section{Le René Guillot qu'on connaît (hors milieux universitaires) et celui qu'on ne connaît pas}

3 La production de récits pour la jeunesse par René Guillot est quantitativement et qualitativement très importante. Entre la fin des années 1930 et sa mort, l'auteur a publié plus de 120 livres pour enfants et adolescents ${ }^{2}$, dont plusieurs ont été réédités. Les récompenses ont également été nombreuses : je signale le Prix du roman d'aventures en 
1946 pour Les Équipages de Peter Hill, le Prix Jeunesse en 1950 pour Sama, prince des éléphants , le Prix Hans Christian Andersen en 1964 pour Le Grand Livre de la Brousse (c'est la plus haute distinction internationale de littérature pour la jeunesse, et Guillot est le premier auteur français à l'avoir reçue).

Guillot a été surnommé « le Kipling saintongeais ». C'est un surnom assez juste, dans la mesure où l'auteur est fort attaché à sa province natale, la Saintonge, qui constitue le décor d'une partie de ses récits, et que l'autre décor de prédilection est représenté par l'Afrique, où les animaux sont protagonistes ou co-protagonistes. La passion pour l'Afrique a, elle aussi, des racines biographiques : à partir de 1925, René Guillot enseigne les mathématiques dans un lycée de Dakar ; il restera au Sénégal pendant 25 ans.

La fortune critique de Guillot est quelque peu paradoxale, dans le sens où il est deux fois ignoré par les milieux universitaires. Il est ignoré en tant qu'écrivain pour les enfants, malgré son succès grand public, parce que la littérature pour la jeunesse y est peu étudiée, et il est inconnu en tant qu'auteur pour adultes, puisque sa production immense et son succès dans le domaine jeunesse ont mis entre parenthèses les romans écrits dans sa première phase créatrice.

\section{Les romans des années 1930}

6 S'interroger sur les «oubliés des années 1930 » implique nécessairement l'étude des processus de réception, et donc de ce mécanisme qui a fait que des écrivains appréciés à une époque - à partir d'une certaine lecture, d'un certain classement - aient été délaissés par la suite. Souvent, le destin des œuvres et des auteurs est lié à la fortune critique de ces classements, qu'il est donc important de mettre au point.

7 Les romans que René Guillot publie dans les années 1930 sont faciles à ranger selon les catégories en vigueur à l'époque : nous avons deux romans régionalistes, ou romans « du terroir » (La Grande Renaude et Taillis), un roman maritime (Vent de Norois) et trois romans coloniaux (les autres). Il s'agit de genres assez en vogue à l'époque : les noms des courants régionaliste et colonial, en particulier, reviennent dans les histoires littéraires des années 1920-19503 , mais aussi par exemple dans les intitulés des sections des comptes rendus dans le Mercure de France, ce qui influence largement la réception des œuvres au moment de leur parution ${ }^{4}$.

Qu'est-ce que le régionalisme et en quoi La Grande Renaude et Taillis sont-ils des romans régionalistes? La littérature régionale est une catégorie vaste, aux contours fluctuants ${ }^{5}$, particulièrement florissante dans l'entre-deux-guerres. Anne-Marie Thiesse a très bien expliqué comment la production littéraire provinciale s'est développée, dès la fin du XIX ${ }^{\mathrm{e}}$ siècle, gagnant progressivement en autonomie ; comment elle a commencé à imposer de nouvelles thématiques, voire de nouvelles esthétiques ${ }^{6}$. Dans les années 1930 , la catégorie est bien établie, reconnue et prisée, si bien que différents éditeurs lancent des collections où l'attention pour tel ou tel territoire est mise en valeur. La réédition chez Arthaud de $L a$ Grande Renaude confirme les classements que j'ai suggérés: dans la page consacrée aux œuvres du même auteur, La Grande Renaude et Taillis figurent parmi les «œuvres du terroir ", alors qu'on retrouve dans un autre ensemble les "œuvres coloniales ». En effet, La Grande Renaude est une histoire dans la veine réalistico-régionaliste, dont le décor est la Saintonge. L'intrigue de Taillis se déroule entre deux pôles géographiques, tous deux localisés en Charente maritime. Les protagonistes des deux récits sont des campagnards 
bien en phase avec leur province, qui se laissent guider par le rythme des saisons et par les travaux que commande la terre.

9 Examinons ces deux romans un peu plus dans le détail. Le décor principal du premier est une ferme dominée par la Renaude, une femme malade, alitée, mais à la volonté de fer. Son fils s'est marié avec Jeanne, une Parisienne, qu'elle méprise et qu'elle nomme "l'Autre » (elle aurait préféré pour son fils une femme du pays). Seule à savoir qu'elle est enceinte, Jeanne abandonne la ferme, laissant son mari triste et inquiet. Si j'acceptais de ne reconnaitre à ce roman que la dimension régionaliste, je terminerais en disant qu'après une série de vicissitudes, la Renaude découvre que Jeanne est malade et prête à accoucher; elle projette alors d'aller chercher l'enfant, qui prouvera que la lignée familiale est bonne et forte. J'ai bien dit « si j'acceptais de ne reconnaître à ce roman que la dimension régionaliste » : l'étiquette peut, à mon sens, être discutée. En effet, La Grande Renaude est aussi un roman psychologique où la dimension de l'altérité est fondamentale. La trame présente un élément qui sera repris dans la plupart des romans de Guillot, à savoir la situation où un personnage se glisse, petit à petit, dans la peau d'un autre, finissant par prendre sa place au milieu de ses proches. Dans La Grande Renaude c'est Suzanne, la sœur aveugle de Jeanne, qui vient à la ferme en son absence et commence à s'habiller et à se coiffer comme elle, altérant les rapports interpersonnels. Ce sera ensuite Eva, «la cousine pauvre », qui essaiera de prendre la place de Jeanne. Le fils de la Renaude est, lui aussi, un personnage de roman psychologique, au bord de la dépression; le romancier s'attarde souvent à décrire «le mal qui le ronge » de l'intérieur. Les actions véritables, dans le récit, sont des épreuves de force psychologiques, ce qui explique d'ailleurs que celle qui "gagne» est la "grande Renaude», qui n'a cependant jamais quitté son lit de malade.

10 Taillis peut être décrit plus ou moins dans les mêmes termes. Le protagoniste est Constantin Geoffroy, un homme assez vieux, déjà frappé par une attaque cérébrale mais qui fait preuve d'une grande force. La situation difficile dans laquelle verse la famille au départ bascule totalement lorsque le fils cadet, qui est un "innocent ", un " pauvre fou ", tue son frère. La famille doit déménager dans une région où une ligne de chemin de fer est en construction, pour laquelle Constantin et son fils se font embaucher. Les pages centrales de Taillis, qui décrivent la vie de la famille dans le nouveau contexte, dans une cité ouvrière bâtie en fonction du chantier, rappellent les romans de la tradition naturaliste ou les romans "prolétariens » qui ont une certaine fortune précisément dans les années 1930. La dimension psychologique, voire psychopathologique, est importante dans Taillis comme dans La Grande Renaude: Constantin, dont le père avait été trouvé pendu à un chêne, se laisse gagner petit à petit par un mal qui le travaille de l'intérieur, et malgré tous les succès escomptés dans sa nouvelle vie il finira par se pendre, lui aussi, " conduit » (c'est un verbe qui revient souvent dans les romans de Guillot) par une force mystérieuse, qui fait penser, d'une part à l'hérédité des Rougon-Macquart, et d'autre part à la dépression en tant que syndrome neurologique telle qu'elle a été décrite surtout au $\mathrm{XX}^{\mathrm{e}}$ siècle.

11 Une première réflexion s'impose : ces deux récits qui, en tant que régionalistes, devraient trouver leur sens ultime dans le décor de l'action, dans la dimension extérieure, sont au contraire centrés sur des dynamiques tout à fait intimistes, psychologiques. On ne peut pas dire que le classement « régionaliste » soit abusif, mais il est sûrement insuffisant.

12 La situation n'est pas entièrement dissemblable dans les autres textes. Bien que de moins en moins lu de nos jours, le roman colonial est un genre aux contours assez définis? 
Histoire... rappelle, par sa structure fondamentale, différents récits coloniaux, dont la tradition est riche, en France surtout à partir du dernier quart du XIX siècle : des soldats blancs, français, s'embarquent, suivent la côte occidentale de l'Afrique et la pénètrent progressivement ${ }^{8}$. Dédié à Robert Delavignette, un haut fonctionnaire spécialiste des questions coloniales, Ras el Gua décroche pour sa part, en 1938, le Grand Prix du roman colonial. Tant dans Histoire... que dans Ras el Gua sont décrits des soldats coloniaux en Afrique, du côté du Mali actuel dans le premier cas, en plein Sahara dans le deuxième. Pas de soldats dans Loaga, mais toujours des Blancs qui travaillent dans des concessions coloniales, cette fois en Côte d'Ivoire. Or, encore une fois, l'étiquette coloniale ne saurait être suffisante. Non seulement la dimension psychologique est tout aussi centrale dans ces romans que dans les deux récits régionalistes (on pourrait même dire qu'un trouble psychologique ou neurologique, avec ses conséquences, constitue le cœur d'Histoire..., de Ras el Gua et de Loaga), mais l'exotisme du roman colonial ${ }^{9}$ se lie dans plus d'un cas avec la dimension régionale, puisque les personnages de Guillot effectuent souvent des passages, parfois des allers-retours, entre l'Afrique lointaine et la province française. Le lieu autre est, dans la plupart des cas, véritablement aliénant, et contribue à créer l'ambiance maladive qui vaudra à certains personnages une sorte de folie.

Les protagonistes de Ras el Gua sont des militaires en poste dans le désert du Sahara ( grosso modo au Niger). La chaleur, l'attente et la solitude les font quelque peu dérailler. Comme dans Taillis, il y a un personnage très simple, analphabète, Péhu, qui a perdu la raison à la suite d'une attaque et qui ne rêve que de sa Marie, qu'il a laissée au pays. Bien qu'absente, « la Marie de Péhu » est la véritable héroïne du récit : tout le monde fantasme sur elle. On découvrira seulement à la fin qu'elle est morte depuis dix ans, que Péhu a contribué lui-même à la noyer (ce qu'il a oublié à la suite du trauma de l'attaque) et que les lettres qu'il croit recevoir d'elle sont écrites successivement par deux de ses camarades. L'un après l'autre, ces derniers prennent la place de Marie à l'insu de Péhu et, conduit en France par Péhu lui-même, l'un d'eux finira par le tuer, dans le vain espoir de prendre sa place à côté de Marie, quitte à découvrir la vérité le lendemain. Le retour en France marque en quelque sorte la fin d'un long mirage.

De même, dans Loaga, le Blanc Mouve, qui ressemble un peu à Péhu et un peu au Barail d' Histoire... (il a lui aussi « le hurlement joyeux d'un nègre ») ${ }^{10}$, a complètement oublié son passé, y compris son identité, à la suite d'un accident en bateau. Employé dans une concession coloniale au cœur de la brousse ivoirienne, il est rejoint par une femme, Anna ; celle-ci fuit son propre passé et essaie de lui faire croire qu'il est la personne avec qui elle a vécu et qui a tué son ancien compagnon. Grâce à ses récits, qui reconstituent la vie qu'elle menait à Nantes, Mouve essaie d'entrer dans la peau de cet inconnu et de se souvenir - en vain. Même s'il avait promis à Anna de retourner en France, après la mort de celle-ci il décide de rester au cœur de l'Afrique; il ignorera toujours son passé.

On voit bien que, dans tous ces cas, le décor colonial fournit le prétexte pour un récit dont le cœur est ailleurs. Ras el Gua se compose en grande partie de dialogues (ce n'est pas pour rien qu'un film en a été tiré en 1947$)^{11}$ et la dynamique qui constitue une constante dans les événements est celle qui fait qu'un homme se mette dans la peau d'un autre, d'abord avec quelque intérêt, puis qu'il soit pris au piège de l'identification. Dans Loaga, l'identification abusive est dictée par la volonté d'un autre personnage, mais le mécanisme est le même. Il faut dire que dans Histoire... et dans Loaga, l'Afrique centrale est un peu plus présente et prégnante que le désert dans Ras el Gua - la biographie de Guillot y étant pour quelque chose. Dans les trois romans, en tout cas, le contexte n'a de sens 
qu'en fonction d'un processus psychologique, de connaissance et de reconnaissance, qui se joue entièrement entre identité et altérité. Or ceci n'est pas la norme dans les récits coloniaux, qui sont bien sûr concernés par les questions d'identité et d'altérité, mais en rapport avec les terres et la population étrangères, et dans des termes culturels : si psychologie il y a, elle est collective. Plus en général, la littérature coloniale est plutôt tendue vers l'extérieur, vers le contexte, qui est décrit, mesuré, compté. L'exotisme d'un Pierre Loti ne se retrouve pas non plus chez Guillot, parce que les drames que vivent les héros de Loti sont intimement liés à la terre qui les accueille, alors que dans les œuvres de l'écrivain saintongeais, ainsi qu'on a pu le constater, les mêmes dynamiques se déclenchent dans la France rurale comme au cœur de l'Afrique. C'est d'ailleurs Guillot luimême qui met l'accent sur l'identité essentielle entre la province et la colonie, et ce depuis la dédicace du premier de ses romans, $L a$ Grande Renaude : «À mon Frère Pic qui saurait situer mes escales africaines sur le chemin de la Varenne et qui comprend pourquoi le Niger peut se perdre et venir se jeter dans la Seugne, entre l'Aubrade et le Port des Groix ». Considérons également que dans l'entre-deux-guerres, aux yeux de beaucoup, la littérature coloniale n'est qu'une variante de la littérature régionaliste : dans les deux cas, il s'agit de mettre en relief un décor périphérique avec un certain parti pris de réalisme.

Un discours à part - mais assimilable à ce qu'on vient d'exposer - doit être fait pour Vent de Norois. On peut tenir le roman maritime pour une sous-catégorie du roman d'aventure, ne s'adressant pas nécessairement à la jeunesse ${ }^{12}$. Le texte de Guillot, dont la trame se déroule pour une part sur la terre ferme et pour une autre à bord, rappelle de très près certains récits de Joseph Conrad comme Typhoon (Typhon) ou The Shadow Line (La Ligne d'ombre). Comme dans Typhoon, un navire doit affronter une tempête, circonstance aggravée, dans Vent de Norois, par le fait qu'une partie du personnel à bord a été payée pour saboter le bateau. Par contre, le sous-commandant Mourne, dont on dit qu'il porte malheur et qu'il perdra la raison à cause de cela, finissant par se faire emporter par la mer, fait penser au vieux capitaine de A Shadow Line maudissant le bateau qui semble effectivement touché par un anathème au moment où une épidémie de fièvre touche la plupart de l'équipage ${ }^{13}$. Mais, dans ce roman comme dans les autres, le décor - ici, la mer et le bateau - est presque symbolique; la description de la tempête donne bien sûr de l'action à la narration, mais ce sont les dynamiques interpersonnelles qui en constituent la charpente : la jalousie du capitaine vis-à-vis de sa femme ; sa douleur lorsqu'il apprend qu'elle ne survivra pas à un accident; une série de malentendus; les mensonges, soupçons et réticences de l'équipage, dont une partie est complice du sabotage.

\section{Relire Histoire d'un Blanc qui s'était fait nègre au spectre de l'altérité}

17 Mais venons-en au roman qui mérite, parmi les autres, la plus grande attention ${ }^{14}$. J'ai souligné que les romans de Guillot infléchissent le genre auxquels ils appartiennent, déplaçant vers la dimension intime, psychologique, le cœur de récits que l'on attendrait tournés vers l'extérieur, et j'ai montré à quel point la dimension de l'altérité est fondamentale dans ce processus. Tout cela apparait, dans Histoire..., au plus haut degré, dans un système complexe et particulièrement savant, qui mériterait d'être redécouvert. 
J'essaie de simplifier le cadre narratif et la trame du roman. Le narrateur, dont on ignore le nom, est en voyage en Afrique subsaharienne. Blessé, il est conduit dans un village et soigné par Barail, un vieil homme qui en est le chef et qui présente les traits d'« un Blanc qui s'est fait nègre ", se réconciliant avec l'Afrique après avoir participé à des razzias dans ces mêmes lieux, quand il faisait partie de l'armée coloniale. Barail décide de raconter son histoire au narrateur, qui en devient donc le porte-parole : la plupart du livre consiste dans l'histoire passée de Barail, racontée à la troisième personne. Ancien enfant trouvé, Barail s'engage dans une troupe coloniale avec deux compagnons : Giraud et Sidoine, qui apparaissent l'un l'opposé de l'autre. Provenant de la campagne saintongeaise, le premier est silencieux, généreux, très bon ; le deuxième, vindicatif et violent, a été marqué par de précédentes missions en Afrique. Tous deux ont un grand ascendant sur Barail, qui épouse tour à tour la volonté de l'un ou de l'autre. En Afrique, pour venger Giraud qu'ils croient assassiné par les hommes d'un village voisin, Sidoine et Barail attaquent le village et ses habitants, réalisant un véritable massacre. Une furie homicide s'empare à nouveau de Sidoine lorsqu'il aperçoit Giraud rentrer au campement, seulement blessé : il se jette sur son camarade et, « conduit » par Sidoine, Barail contribue à tuer Giraud pour de bon. Rendu à la vie civile, Barail se rend dans la ferme où vivait son ami et il finit par prendre sa place dans sa famille, dans sa maison, avec sa compagne. De temps en temps, la pensée de Sidoine et de l'Afrique le regagne. Après avoir pris part à la Grande Guerre, il obtient de se rengager dans son grade et, sans plus jamais retourner à la ferme de Giraud, fait différentes campagnes coloniales. Avec l'argent mis de côté, il demande à l'administration coloniale la concession d'une portion de terre, celle-là même où surgissait le village qu'il a détruit avec Sidoine et où il dit avoir enterré Giraud. Malgré d'innombrables adversités, il bâtit le village à nouveau, s'y installe et cherche à se réconcilier avec la terre qu'il a " violée » auparavant. Une fois le narrateur guéri, Barail découvre un autre blessé dans la brousse et se persuade qu'il est le fils de Sidoine. Il l'assiste jusqu'à sa mort et mourra luimême peu de temps après. Lorsqu'ils creusent une fosse pour ensevelir Barail entre les restes de Giraud et le corps de cet homme censé être le fils de Sidoine, les villageois s'aperçoivent que la tombe de Giraud est vide. Le narrateur ne poursuit pas son voyage en Afrique et rentre en France, effaré : il n'a plus aucune certitude sur ce qu'il a vu et entendu raconter, mais il sent la nécessité de raconter, à son tour, cette même histoire.

En dépit de cette trame complexe, les décors principaux de l'action ne sont que deux : l'Afrique centrale et la Saintonge. Les chapitres qui voient Barail prendre la place de Giraud à la ferme constituent un micro-texte régionaliste enchâssé dans un roman colonial.

Le poids que prend la dimension de l'altérité dans la définition des personnages et dans la construction de l'intrigue des autres romans de Guillot devient écrasant dans Histoire.... Dès les premières lignes, le narrateur prévient qu'il est là pour raconter l'histoire d'un autre; cet autre est un homme qui ne vit que dans l'altérité (Blanc au milieu des Noirs, et tel un Noir aux yeux des Blancs) et qui y succombe suite à un voyage aliénant en Afrique. Or le pouvoir aliénant de l'Afrique, surtout aux alentours de l'« époque des Empires", était hors de doute pour la plupart des Européens. Pénétrer le continent mystérieux, c'était sortir des frontières rassurantes de «la » civilisation et accéder à une sorte de monde à l'envers. La particularité d'un personnage comme Barail est qu'il n'a pas attendu d'arriver dans le continent mystérieux pour être habité par des formes d'altérité : doté d'une personnalité assez faible, il vivait pour ainsi dire déjà la vie des autres. Cette tendance atteint des niveaux pathologiques: Barail devient d'abord Giraud, quitte à se 
laisser encore une fois conquérir par l'esprit de Sidoine, notamment pendant la guerre. Il faudra attendre son retour en Afrique pour que Barail se mette au service des autres, au pluriel, se libérant peu à peu - mais jamais complètement - de ces présences qui l'habitent.

Le passage à l'altérité est fondamental pour comprendre non seulement les personnages d'Histoire..., mais la construction du texte dans son ensemble. En effet, l'un des intérêts principaux du roman réside dans le dialogue qu'il instaure avec plusieurs autres textes.

Tout d'abord, Histoire... peut être considérée comme l'une des premières réécritures en français de Heart of Darkness (Au cœur des ténèbres). Publié en revue en 1899 et en volume en 1902, le chef-d'œuvre de Joseph Conrad a connu différentes réécritures, sur l'étendue de plus un siècle ${ }^{15}$, et quelque étrange que cela puisse paraître, les premiers exemples en français datent précisément du début des années $1930^{16}$. Avant de dire en quoi Histoire... est une reprise de Heart of Darkness, il convient de rappeler que le roman de Conrad reprend à son tour la matrice d'un texte fondateur de la tradition coloniale internationale, à savoir Through the Dark Continent (À travers le continent mystérieux) de l'explorateur Henry Morton Stanley, parti à la recherche du Dr Livingstone. La matrice stanleyienne peut être synthétisée comme suit : un homme blanc pénètre dans le cœur de l'Afrique à la recherche d'un autre Blanc qui a été englouti par son mystère, le trouve et tous deux rentrent dans leur pays. La matrice conradienne approfondit la dimension intérieure du processus : un homme blanc se rend au cœur de l'Afrique et est conquis par une sorte de folie; un autre Blanc va le chercher, se laisse quelque peu contaminer par cette même folie (qui n'est autre qu'une forme de connaissance), assiste à la mort du premier et rentre en Europe où il livre le récit onirique de son voyage. On conviendra qu' Histoire... se rapproche beaucoup plus de ce deuxième modèle, où la dimension extérieure est fortement concurrencée par la dimension intime. Le voyage mène à la connaissance, mais l'Afrique n'en est plus l'objet principal : elle est seulement le cadre le plus propice à ce processus heuristique. L'intériorisation $d u$ processus de découverte est particulièrement complexe dans Histoire..., puisqu'elle est l'affaire de différents personnages : d'abord Sidoine, puis Barail, enfin le narrateur lui-même.

Au-delà du décor et de ce processus aliénant à la base des deux récits, les renvois de Guillot au texte de Conrad sont nombreux. Comme Kurtz, Barail vit au cœur de la brousse, entouré par des Africains; ce sont deux personnages qui fascinent, qui ont conquis les locaux par leurs actions mais, avant tout, par leur parole : à l'éloquence légendaire de Kurtz correspond la " palabre » de Barail, qui tend à monologuer comme son antécédent anglophone ${ }^{17}$. Cependant, la figure de Barail ne peut se réduire à celle de Kurtz. Notons, entre autres, que si Barail s'est fait engloutir par les ténèbres lors de son premier voyage en Afrique, comme Kurtz, il y est retourné pour expier sa faute et pour se réconcilier avec cette terre qu'il entend désormais respecter et même servir. Il y a, dans Le Blanc qui s'était fait nègre, une dimension spirituelle, voire mystique, qui est absente de Heart of Darkness, et qui trouve un appui important dans une autre relation intertextuelle, avec le conte flaubertien de La Légende de Saint-Julien l'hospitalier.

Barail est, avant tout, l'hôte du narrateur, celui qui lui offre l'hospitalité. Et il est présenté comme « un primitif ardent, cruel, et mystique... » (je souligne). C'est la même tension qui caractérise Julien: la cruauté et la bonté, la violence et l'élan mystique, avec cette différence que, chez Guillot, ces deux tendances opposées s'incarnent dans deux personnages différents, Giraud et Sidoine, avant d'être intériorisées par le protagoniste. Comme Julien, Barail se distingue par des excès de férocité et se rattrape ensuite par un 
service rendu dans l'abnégation ${ }^{18}$. La fatalité de matrice religieuse qui sous-tend $\mathrm{La}$ Légende de Saint-Julien l'hospitalier est absente d'Histoire..., mais elle trouve une sorte d'équivalent dans la tendance qu'a Barail de se faire guider par les autres. Si Barail devient un assassin comme Julien, c'est qu'il a été conquis par la sauvagerie de Sidoine et « conduit » par sa volonté. Ensuite, pour expier son mal, il retourne en Afrique où il s'évertue au service des indigènes, devenant le plus miséreux d'entre eux. C'est seulement dans un moment ultérieur, comme Julien après le meurtre de ses parents, que Barail sera en quelque sorte " condamné » à raconter son histoire - et le narrateur après lui. Et là, le roman de Guillot montre les mêmes dettes que le conte flaubertien vis-à-vis d'un autre classique de la littérature, à savoir The Rime of the Ancient Mariner. Dans la célèbre ballade de Samuel T. Coleridge, un homme (le marin) est maudit après avoir tué un albatros, ce qui entraîne la mort de tout son équipage ${ }^{19}$. Il se rattrapera ensuite, mais il sera tout de même condamné à parcourir le monde sans fin, à la manière du juif errant, contraint à raconter son histoire en guise d'exemplum.

Quel sens pouvons-nous attribuer à ce dialogue intertextuel ? Il faut d'abord noter que le cadre classique du roman colonial est largement dépassé. L'autre remarque qui s'impose est que la nécessité d'expiation qu'indiquent les traits communs avec La Légende de SaintJulien l'hospitalier suggère un jugement sévère vis-à-vis des abus perpétrés par l'armée coloniale. La seule issue à l'horreur est, pour le Kurtz de Conrad, la conscience de celle-ci, puis la mort; par l'intermédiaire de Barail, Guillot montre, de son côté, une autre voie possible, où l'Afrique devient un lieu accueillant pour les Blancs aussi, à condition qu'ils vivent en égaux avec les autochtones.

Le texte de Guillot dialogue aussi avec d'autres disciplines. Histoire... est doublement un « voyage au bout de la nuit $»^{20}$ : c'est un voyage dans l'Afrique mystérieuse, voire dans le noir de la conscience coloniale tachée du sang de ses victimes, et en même temps un voyage au bout du gouffre de la psyché humaine. Dans Histoire..., l'allusion aux théories freudiennes est évidente. Pour Guillot, proposer un voyage dans la psyché à travers la psychologie freudienne est une autre manière d'approfondir la question de l'altérité, qui lui est si chère. En effet, Freud a été le premier à insister sur la dissociation du moi. Si, dans un cadre mystique ou moral, Giraud et Sidoine représentent le Bien et le Mal, la vertu et le vice, dans un cadre psychanalytique ils font évidemment penser au surmoi et au ça, d'autant plus que Barail intériorise les traits de l'un et de l'autre.

D'ailleurs, il faut savoir que, directement influencé par la lecture des récits de voyage des explorateurs coloniaux (Stanley en premier), Sigmund Freud s'est servi, à plusieurs reprises, de la métaphore de l'Inner Africa, l'«Afrique intérieure", pour désigner le royaume de l'inconscient ${ }^{21}$. Ce n'est pas tout: il a également théorisé la tripartition de l'âme entre les royaumes des Noirs, des métis et des Blancs ${ }^{22}$. C'est en quelque sorte ce que l'on trouve dans le récit de Guillot : le royaume du Noir d'un côté (Sidoine, le mal) ; le royaume du Blanc de l'autre (Giraud, le bien); et, au milieu, ce «Blanc qui s'était fait nègre ", cet espèce de métis - pour reprendre, à nos fins, la métaphore freudienne -, ballotté entre un royaume et l'autre. Une remarque importante: le royaume du Noir et celui du Blanc ne correspondent pas forcément, chez Guillot, à l'Afrique et à l'Europe géographiques et n'impliquent nullement la méchanceté ou la bonté de leurs populations respectives. Il est vrai que Sidoine se déchaîne surtout en Afrique et que Giraud est solidement enraciné en Europe, mais Barail prouve qu'on peut incarner les idéaux de Giraud même au cœur des ténèbres et que les Noirs, eux, n'ont rien à voir avec les 
ténèbres qui habitent l'âme, au point que c'est parmi eux que Barail essaie de purifier la sienne.

\section{Les raisons d'un oubli}

Avec les pages qui précèdent j'espère avoir démontré au moins deux choses : primo, que les romans que Guillot a publiés dans les années 1930 ne sauraient se réduire aux classements qui étaient admis au moment de leur publication, et dans lesquels ils sont restés, faute de relectures; secundo, que certains d'entre eux mériteraient d'être redécouverts, surtout Histoire..., d'autant plus que la lecture que peut faire de ce récit un lecteur du XXI ${ }^{\mathrm{e}}$ siècle s'est enrichie d'éléments qui échappaient sans doute au public des années 1930.

ces romans ont sombré dans l'oubli, c'est à mon avis en partie pour des raisons qui sont propres à Guillot et en partie pour d'autres qui sont communes à d'autres auteurs des années 1930.

Parmi les causes qui ne concernent que Guillot, la première est la dispersion éditoriale : La Grande Renaude et Loaga paraissent au Sénégal, Ras el Gua et Vent de Norois au Maroc; Histoire... et Taillis à Paris, par les soins de Rieder, une maison d'édition rachetée par les PUF à la veille de la Seconde Guerre mondiale, dont les archives ont disparu ${ }^{23}$. Mais surtout, Guillot s'est, après la période qui nous concerne, spécialisé dans la littérature pour la jeunesse, ce qui a contribué à jeter dans l'ombre sa production de la première heure, quantitativement tout à fait négligeable par rapport aux dizaines et dizaines de récits destinés aux enfants. Le fait d'être couronné en 1946 par le prix Andersen semble définir, une fois pour toutes, l'appartenance de l'écrivain à un domaine au détriment des autres. Pour finir, Guillot connaît le succès dans l'après-guerre, et est dès lors reconnu comme un écrivain des années 1950 et 1960 : les romans des années 1930 semblent vraiment une parenthèse lointaine.

Parmi les raisons de l'oubli qui sont communes à d'autres auteurs de la même période, il faut certainement mentionner l'appartenance des romans cités à des courants qui, depuis, ont disparu ou ont été évalués négativement.

Le régionalisme est un mouvement aux contours peu nets qui s'est vu reconnaître une certaine visibilité dans la première moitié du $\mathrm{XX}^{\mathrm{e}}$ siècle, mais auquel pour le reste il n'a jamais été attribué beaucoup de légitimité. L'adjectif "régionaliste» (un peu comme, mutatis mutandis, "populiste ») est même tenu par certains pour quelque chose de limitatif, sinon de négatif. Plus généralement, les courants qui se sont affirmés à partir des années 1950 - et je pense surtout à la nébuleuse Nouveau Roman - ont jugé de manière très sévère ce qu'on pourrait appeler le " nouveau réalisme » de l'entre-deuxguerres (récits de guerre, régionalisme, écriture du terroir, populisme, littérature prolétarienne...), parce qu'ils y ont reconnu des parentés trop étroites avec le réalisme du $\mathrm{XIX}^{\mathrm{e}}$ siècle et avec le naturalisme ${ }^{24}$, c'est-à-dire avec le passé qu'ils voulaient le plus condamner, et parce qu'ils voulaient s'affirmer, eux, comme les nouveaux réalistes du XX e siècle. Comme c'est toujours le cas, l'histoire littéraire se construit à rebours : l'après décide des valeurs de l'avant, et comme en histoire tout court, ce sont les vainqueurs qui écrivent le passé, décidant ce qui mérite d'être retenu et transmettant l'information aux générations suivantes. Pas de surprise, donc, à ce que même aujourd'hui, aux yeux de tant 
de monde, l'entre-deux-guerres soit une période essentiellement dominée par Proust et par le surréalisme.

Le roman colonial fait, quant à lui, référence à un monde qui a disparu. Les guerres pour l'indépendance des anciennes colonies marquent, toujours dans les années 1950- et 1960, la fin d'une époque dont les reflets littéraires s'épuisaient déjà depuis un certain temps. Aujourd'hui, tous les essais portant sur la littérature coloniale débutent par des justifications, comme s'il était réprouvable de se concentrer sur un tel objet - alors qu'une analyse plus fouillée de ces textes (répétitifs dans l'ensemble, on ne dira pas le contraire, et souvent médiocres) nous en dirait long sur les imaginaires liés à l'ailleurs que nous n'avons pas cessé de partager. Les récits coloniaux qui ont survécu, estimés (à juste titre) par la critique, l'ont fait au prix de renier quelque peu leur contexte original. Heart of Darkness en est peut-être le meilleur exemple : longtemps, grâce aussi à l'analyse de Todorov, la "connaissance du vide» a été privilégiée au détriment d'une lecture historicisante ${ }^{25}$. D'où l'attaque célèbre de Chinua Achebe ${ }^{26}$ et une certaine réaction, essentiellement limitée au domaine anglophone où, à la suite des processus des indépendances des territoires africains, se sont multipliées les réécritures du chefd'œuvre conradien, lues et très souvent écrites dans une optique postcoloniale. Intéressante en elle-même, cette optique a pour effet d'aplatir quelque peu la complexité du texte de départ (tout comme la lecture de Todorov mettait en sourdine la dimension historique du texte). Comme ces réécritures sont étudiées essentiellement par des spécialistes de littérature anglophone, Histoire... est resté aux marges de ce processus de redécouverte.

D'une manière ou d'une autre, les romans de Guillot des années 1930 souffrent du contexte qui les a vus naitre, de l'alternance des courants critiques et de la fortune de ceux-ci. Seule une relecture à la fois historique et analytique pourrait les sortir de cet oubli ; une relecture qui situe leur naissance dans un contexte précis mais qui étudie les textes au-delà des limites étriquées des catégories dans lesquelles on les a d'abord placés. S'il est tant d'« oubliés des années 1930 ", c'est qu'il y a un véritable problème lié aux classements de cette période et au sort ingrat que leur a réservé notamment la critique des années 1950-1970, dont nous sommes encore les héritiers. Car si les romans des années 1930 doivent être replacés dans leur contexte, la même chose vaut pour la critique qui les a condamnés à l'oubli.

\section{NOTES}

1. La Grande Renaude, Dakar, Ars Africae, 1929 ; Histoire d'un Blanc qui s'était fait nègre, Paris, Rieder, « Prosateurs français contemporains ", 1932 ; Taillis, ibid., 1934 ; Ras el Gua, poste du Sud. Roman des sables, Casablanca, Les Éditions du Moghreb, 1936 ; Vent de Norois, ibid., 1938 ; Loaga, Dakar, Ars Africae, 1941. Histoire d'un Blanc qui s'était fait nègre a reparu en 1946 chez SFELT sous le titre Le Blanc qui s'était fait nègre et a fait l'objet, en 2013, d'une réédition par mes soins dans la collection «Autrement mêmes » de L'Harmattan; c'est à cette réédition que je renvoie dans cet article. La Grande Renaude a également reparu, en 1946, chez Arthaud. 
2. Pour la bibliographie complète de Guillot, cf. S. Drey, René Guillot:le Kipling saintongeais. Biographie d'un écrivain charentais, Rochefort, La Malle aux Livres, "Mémoires charentaises ", 2000, p. 245. Malheureusement, l'intérêt de ce livre est purement documentaire, et les données sont même parfois inexactes.

3. Cf. par exemple A. Billy, La littérature française contemporaine : poésie, roman, idées, Paris, Armand Colin, 1927 et R. Lalou, Le roman français depuis 1900, Paris, PUF, « Que sais-je », 1957.

4. Le Mercure de France divisait en effet ses comptes rendus en sections appelées "romans historiques », « romans féminins ", « romans exotiques et coloniaux », etc.

5. Cf. A. Boivin, H.-J. Lüsebrinck, J. Walter (dir.), Régionalismes littéraires et artistiques comparés. Québec/Canada - Europe, Nancy, Presses universitaires de Nancy, «Questions de communication, série actes », 2015, en particulier A. Von Ungern-Sternberg, "Qu'est-ce que le régionalisme littéraire? ", p. 19-30.

6. A.-M. Thiesse, Écrire la France. Le mouvement littéraire régionaliste de langue française entre la Belle Époque et la Libération, Paris, PUF, « Ethnologies », 1991.

7. Cf. Le Roman colonial, Itinéraires et contacts de cultures, 7, 1987, et Le Roman colonial, suite, 12, 1990 ;

P. Halen, Le Petit Belge avait vu grand: une littérature coloniale, Bruxelles, Labor, «Archives du futur », 1993 ; J.-M. Seillan, Aux sources du roman colonial (1863-1914) : l'Afrique à la fin du XIX siècle, Paris, Karthala, «Lettres du Sud», 2006 ; J. Riesz, De la littérature coloniale à la littérature africaine : prétextes, contextes, intertextes, ibid., 2007.

8. Cf. F. Moretti, Atlante del romanzo europeo 1800-1900, Torino, Einaudi, 1997, p.62, et M. C. Gnocchi, "Attraverso il continente nero su bianco», dans L.Acquarelli, M. Baraldi, M. C. Gnocchi, V. Russo, Tenebre bianche. Immaginari coloniali fin de siècle, Reggio Emilia, Diabasis, «Passages », 2008, p. 125-169.

9. J'emploie ici le terme « exotisme » au sens large. Denise Brahimi a bien tracé la différence qui sépare les romans exotiques des romans coloniaux et, selon ses définitions, les romans de Guillot sont tout à fait coloniaux. Cf. D. Brahimi, «Pierre Loti, du roman exotique au roman colonial », dans Le Roman colonial, cit., p. 15-27.

10. R. Guillot, Loaga, cit., p. 35.

11. Intitulé Fort de la solitude et dirigé par Robert Vernay.

12. O. Gannier, Le Roman maritime. Émergence d'un genre en Occident, Paris, Presses de l'Université Paris-Sorbonne, « Imago Mundi », 2011.

13. Ce fait est particulièrement intéressant du moment que Histoire... se présente comme une réécriture de Heart of Darkness (Au cœur des ténèbres) du même Conrad (cf. infra).

14. Dans ce paragraphe, je reprends certains éléments de l'introduction que j'ai rédigée pour la réédition du livre pour l'Harmattan (op. cit.).

15. Cf. R. Farm, Colonial and postcolonial rewritings of Heart of darkness : a century of dialogue with Joseph Conrad, Boca Raton, Dissertation.com, 2005.

16. Les éditions originales des textes de Conrad sont signalées en France dès le tournant du siècle, mais c'est seulement en 1924 que paraît, dans un numéro de la Nouvelle Revue française, la première partie de la traduction de Heart of Darkness, qui sera complétée dans les livraisons successives; le volume est publié l'année suivante: Jeunesse suivi de Cour des ténèbres, tr. fr. de G. J. Aubry et A. Ruyters, Paris, Gallimard, 1925.

17. Pour une analyse plus fouillée des convergences existant entre ces deux textes, cf. l'introduction à la réédition du livre que j'ai déjà citée.

18. Idem pour les convergences textuelles entre Histoire... et le conte flaubertien.

19. Ce qui n'est pas sans rappeler la situation qui se crée dans A Shadow Line, dont j'ai évoqué les convergences avec Vent de Norois.

20. Je ne cite pas par hasard le roman de Céline, qui paraît en 1932 comme le roman de Guillot et dont le volet africain constitue, lui aussi, une réécriture de Heart of Darkness. 
21. Cf. U. Link-Heer, «L'Afrique comme métaphore », dans P. Samba Diop, H.-J. Lüsebrink (dir.), Littératures et sociétés africaines, Mélanges offerts à J. Riesz à l'occasion de son soixantième anniversaire, Tübingen, Gunter Narr Verlag, 2001, p. 3.

22. Ibid., p. 11.

23. Les éditions Rieder publiaient depuis une dizaine d'années, dans la collection « Prosateurs français contemporains ", des récits d'auteurs français et francophones, de préférence résidant soit dans la province française, soit dans différents pays francophones européens ou extraeuropéens ; en publiant Guillot, Rieder choisit pour ainsi dire la province et l'étranger à la fois. Cf. M. C. Gnocchi, Le Parti pris des périphéries. Les «Prosateurs français contemporains " des éditions Rieder (1921-1939), Bruxelles, Le Cri-CIEL, 2007.

24. Cf. Ph. Baudorre, «Zola, 1929-1935 ou les ambiguïtés d'un retour de Zola », dans Les Cahiers naturalistes, 65, 37e année, p. 7-23.

25. T. Todorov, Connaissance du vide: Cœur des ténèbres, dans Id., Poétique de la prose suivi de Nouvelles recherches sur le récit, Paris, Seuil, 1980.

26. Ch. Achebe, "An image of Africa : Racism in Conrad's Heart of Darkness ", dans Massachusetts Review, 18, 1977, p. 782-794, repris dans Hopes and Impediments, London, Heinemann, 1988, p. 1-20.

\section{RÉSUMÉS}

René Guillot (1900-1969) est un auteur renommé dans le domaine de la littérature pour la jeunesse, ou qui l'a été en tout cas, surtout dans les années 1960. Ce qui est moins connu, c'est qu'au début de sa carrière, il a écrit des romans non destinés aux enfants, qui couvrent presque exactement la période des années 1930. Cet article propose des pistes de lecture pour ces romans et suggère pourquoi on aurait intérêt à les reprendre en considération. Une attention particulière est accordée au récit le plus complexe, Histoire d'un Blanc qui s'était fait nègre (1932).

\section{INDEX}

Mots-clés : Oubliés, Guillot (René), régionalisme, littérature coloniale, Heart of Darkness, altérité, intertextualité, années Trente 